\title{
Structure of Si(111)-In Nanowires Determined from the Midinfrared Optical Response
}

\author{
S. Chandola, ${ }^{1,2}$ K. Hinrichs, ${ }^{1}$ M. Gensch, ${ }^{1}$ N. Esser, ${ }^{1}$ S. Wippermann, ${ }^{3}$ W. G. Schmidt, ${ }^{3}$ F. Bechstedt, ${ }^{4}$ \\ K. Fleischer, ${ }^{2}$ and J. F. McGilp ${ }^{2, *}$ \\ ${ }^{1}$ ISAS-Institute for Analytical Sciences, Department Berlin, Albert-Einstein Str. 9, 12489 Berlin, Germany \\ ${ }^{2}$ School of Physics, Trinity College Dublin, Dublin 2, Ireland \\ ${ }^{3}$ Lehrstuhl für Theoretische Physik, Universität Paderborn, 33095 Paderborn, Germany \\ ${ }^{4}$ Institut für Festkörpertheorie und optik, Friedrich-Schiller-Universität, Max-Wien-Platz, 1, 07743 Jena, Germany
}

(Received 3 March 2009; published 5 June 2009)

\begin{abstract}
The anisotropic optical response of $\mathrm{Si}(111)-(4 \times 1) /(8 \times 2)$-In in the midinfrared, where ab initio studies predict significant changes in the band structure between competing models of this important quasi-1D system, has been measured using infrared spectroscopic ellipsometry (IRSE) and reflection anisotropy spectroscopy (RAS). Both IRSE and RAS of the $(8 \times 2)$ phase show that the anisotropic Drude tail of the $(4 \times 1)$ phase is replaced by two peaks at 0.50 and $0.72 \mathrm{eV}$, which appear in ab initio optical response calculations for the hexagon model of the $(8 \times 2)$ structure, but not the trimer model.
\end{abstract}

PACS numbers: 73.20.At, 78.68.+m

One-dimensional (1D) electronic systems are attracting considerable interest for both fundamental and technological reasons. Fascinating phenomena, such as spin-charge separation [1], and charge-density wave (CDW) formation due the Peierls instability may appear, while quasi-1D structures are being investigated as atomic-scale interconnects in devices. Such structures can be grown by selfassembly on $\mathrm{Si}$ surfaces [2], where the existence of an energy gap prevents the coupling of the electronic states of the nanostructure with the substrate in the vicinity of the Fermi level, thus preserving the quasi-1D character of the nanostructure states.

An intensively studied model system is the ordered array of In nanowires that self-assembles at the $\mathrm{Si}(111)$ surface to form the $\mathrm{Si}(111)-(4 \times 1)$-In phase, which has quasi-1D metallic properties at room temperature (RT). Results from many different experiments, as well as $a b$ initio band structure calculations, agree that the structure is metallic, with the In atoms forming two parallel zigzag chains, which are separated by zigzag $\mathrm{Si}$ chains that resemble the $\pi$-bonded chains of $\mathrm{Si}(111)-(2 \times 1)$ [3-14]. The structure has three quasi-1D surface state bands, which disperse strongly and cross the Fermi level along the chain direction, and show only a weak dispersion perpendicular to the chain.

Yeom et al. [15] reported that $\mathrm{Si}(111)-(4 \times 1)-$ In undergoes a reversible phase transition below $120 \mathrm{~K}$ to an $(8 \times$ 2) structure, with a strong reduction of the density of states at the Fermi energy. From photoemission and scanning tunneling microscopy (STM) studies they concluded that this phase formed a 1D CDW system driven by a Peierls instability. These intriguing results provoked much experimental and theoretical work. However, the origin of the phase transition remains controversial. The phase transition cannot be based on a simple CDW model because only one of the metallic bands nests properly [3,15-19]. A triple-band Peierls instability has been suggested, where an interband charge transfer modifies the Fermi surface to improve nesting [17], while a periodic lattice distortion that lowers the energy has also been suggested $[6,9,10,20]$. Recently, it has been proposed that, while the Peierls transition is important, other structural transitions are cooperatively involved [21].

Not only the mechanism of the $(4 \times 1) \rightarrow(8 \times 2)$ phase transition, but also the LT ground-state and its properties remain controversial. While the RT $(4 \times 1)$ phase is a quasi-1D metal $[12,16,22]$, it has been variously suggested that the LT $(8 \times 2)$ phase is metallic, but with a lower density of states at the Fermi level [19,20,23], semimetallic [19], and semiconducting with a fundamental energy gap of $0.1-0.3 \mathrm{eV}$ [15-18,21]. Most $a b$ initio calculations predict the nanowire ground state to be characterized by the formation of In trimers (cf. Fig. 1) and find no gap opening $[6,7,9,10]$. Recently, however, a structure with In hexagons, resulting from shear distortions in neighboring chains (Fig. 1), has been predicted, which is semiconduct-

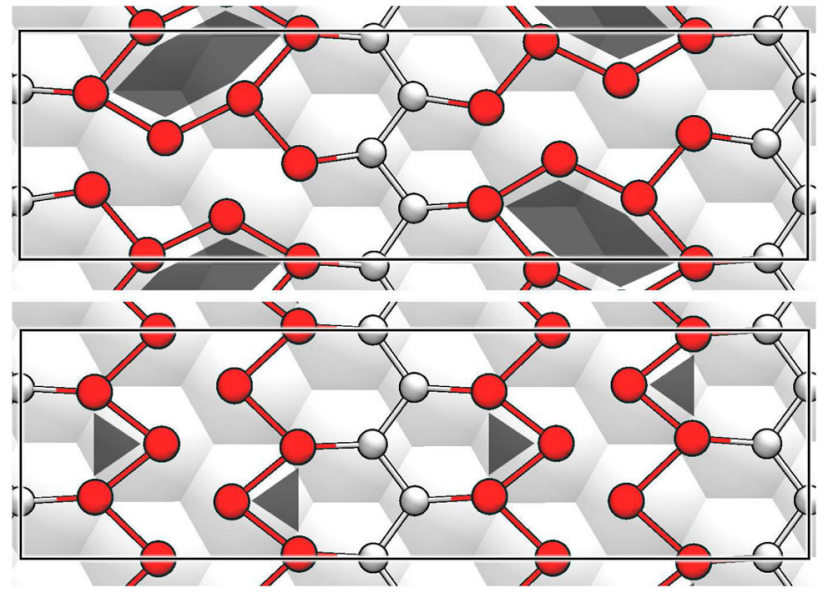

FIG. 1 (color online). Schematic top views of the hexagon and trimer model of the LT Si(111)-In nanowire array. 
ing [24]. In Ref. [24] it was argued that the metallic RT phase arises from dynamic fluctuations between degenerate ground states, but recent photoemission [21] and Raman spectroscopy [11] results have cast doubt on this model. Total-energy calculations [13] of the hexagon and the trimer model for the LT phase concluded that an unambiguous identification of the internal structure of the ground state on energetic arguments is problematic. The energy differences between the competing structures are very small and depend on the approximations made in the calculations, e.g., the treatment of the In $4 d$ states, and exchange and correlation effects. Cho and Lee [25] state that the hexagon model is not stable, but the model has been supported recently by positron diffraction studies [26].

Given these ambiguities, optical spectroscopic information from a new spectral region may be helpful. The small differences in geometry of the trimer and hexagon model lead to significant changes in the band structure near the Fermi level [13,24]. Direct optical transitions in this energy region, as probed by infrared spectroscopic ellipsometry (IRSE) and reflection anisotropy spectroscopy (RAS), are expected to be very sensitive to such changes.

In this Letter, we report the first measurements of optical excitation around the Fermi level for this system, in the mid-IR spectral range from 0.31 to $0.99 \mathrm{eV}$. We show that IRSE has submonolayer sensitivity in this spectral region and can discriminate between metallic and insulating electronic structures. In addition, the comparison of measured data with $a b$ initio calculations provides strong evidence that excludes the trimer model and supports the hexagon model of the In nanowire ground state.

Vicinal, wedged $n$-type $\operatorname{Si}(111)$ samples offcut by $1^{\circ}$ towards the $\left[\begin{array}{lll}1 & 1 & 2\end{array}\right]$ direction were cleaned by heat treatment under ultrahigh vacuum (UHV), and a predominantly single domain $\mathrm{Si}(111)-(4 \times 1)$-In structure was grown by depositing In on the clean Si surface at $670 \mathrm{~K}$. The surface order and the phase transition were monitored in situ with RAS, which has been shown to be very useful in monitoring the surface reconstructions of $\mathrm{Si}(111)$ [27]. The RAS measurements indicated that the dominant domain occupied about $80 \%$ of the surface. Further experimental details are reported elsewhere [28]. IRSE measurements [29,30] were made under UHV conditions using a photometric ellipsometer attached to a Bruker Vertex 70 series Fourier Transform Interferometer with a spectral resolution of $16 \mathrm{~cm}^{-1}$. Ellipsometry measures the change of the polarization state upon reflection or transmission from the sample surface. The measurements were performed in reflection at $65^{\circ}$ angle of incidence and the polarization state of the reflected radiation was analyzed [30]. The ellipsometric parameters, $\psi$ and $\Delta$, are related to the complex reflection coefficients for polarization perpendicular and parallel to the plane of incidence, $r_{s}$ and $r_{p}$, by $r_{p} / r_{s}=$ $\tan \psi \exp (i \Delta)$. In this Letter, only $\tan \psi$ was measured, as a sufficiently sensitive retarder was not available to measure the phase difference $\Delta$. Measurements of the clean Si surface at room temperature (RT) and at low temperature (LT) were used to normalize the In results presented in Figs. 2 and 3. A gap in the spectrum between $3600-3900 \mathrm{~cm}^{-1}$ is shown when the vibrational bands of atmospheric $\mathrm{H}_{2} \mathrm{O}$ are significant. Measurements were made with the plane of incidence parallel to the In chains, along [110], and perpendicular to the chains, along [ $\left.\begin{array}{lll}\overline{1} & 1 & 2\end{array}\right]$. These directions refer to the majority domain, but $20 \%$ of the surface is covered by two minority domains, rotated by $120^{\circ}$.

Figure 2 shows normalized IRSE spectra for the RT $(4 \times 1)$-In and LT $(8 \times 2)$-In phases, in the two orthogonal directions (the clean Si RT and LT spectra are shown at the bottom of the figure). The optical response is clearly anisotropic, particularly when allowing for the parallel contribution from the minority domains. There is a dramatic difference between the $(4 \times 1)$ and $(8 \times 2)$ phases in Fig. 2. The RT In-Si $(4 \times 1)$ metallic phase shows a low energy Drude-like tail and no interband transitions below $1 \mathrm{eV}$. This is in contrast to the behavior above $1 \mathrm{eV}$, where the response of the $(4 \times 1)$ structure is dominated by interband transitions [31,32]. The larger Drude-like response parallel to the chains arises from their highly anisotropic polarizabilities [27] and is consistent with the anisotropic conductivity of this surface, as measured by four-point probe STM $[12,16]$. The spectra show that IRSE can detect anisotropic metallic behavior at submonolayer coverages.

Three main observations can be made about the LT $(8 \times$ 2) spectra: (i) the spectral weight at low energy reduces dramatically, (ii) two peaks appear at $4015 \pm 10 \mathrm{~cm}^{-1}$ $(0.498 \pm 0.002 \mathrm{eV})$ and $5800 \pm 10 \mathrm{~cm}^{-1} \quad(0.719 \pm$ $0.002 \mathrm{eV}$ ), and (iii) a very small feature appears, centered at $2850 \pm 100 \mathrm{~cm}^{-1}(0.35 \pm 0.01 \mathrm{eV})$. This region is en-

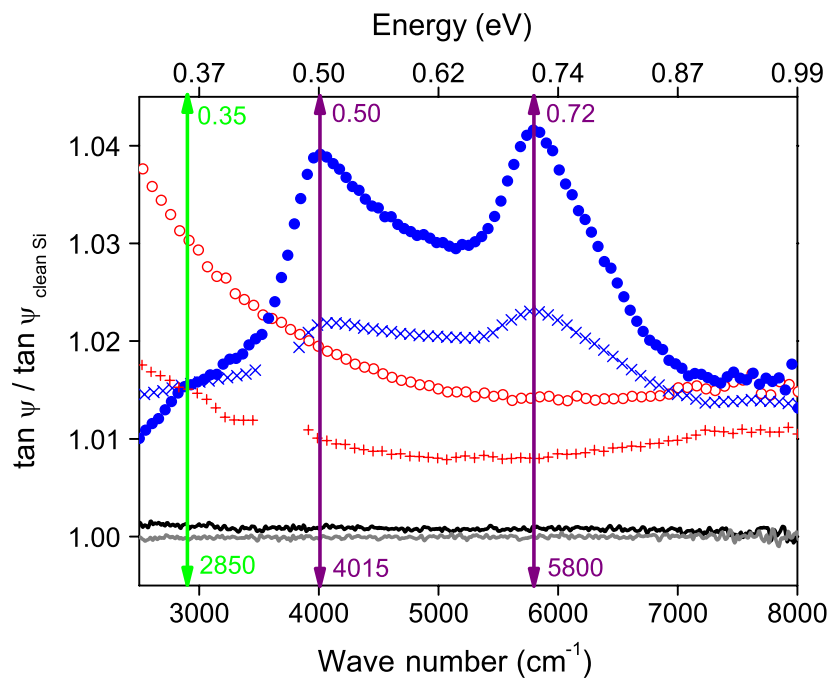

FIG. 2 (color online). IRSE spectra of the RT and LT In-Si phases, for planes of incidence parallel and perpendicular to the In chains: $(4 \times 1)$ parallel $(0),(4 \times 1)$ perpendicular $(+),(8 \times$ 2) parallel $(\bullet),(8 \times 2)$ perpendicular $(\times)$. The clean $\mathrm{Si}$ spectra at 300 and $70 \mathrm{~K}$ are shown at the bottom. 
larged in Fig. 3, where a linearly increasing background has been subtracted in order to emphasize the peak, which grows in intensity when the surface is cooled. Previous high-resolution electron energy loss (HREELS) measurements of the $(8 \times 2)$ phase, which showed three very

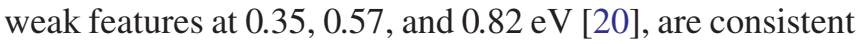
with the results reported here, given the poorer SNR of HREELS at these higher energies. The small feature at $0.35 \pm 0.01 \mathrm{eV}$ agrees within error with the fundamental gap of $0.30 \pm 0.05 \mathrm{eV}$ measured by STM [16]. The removal of the low energy tail in the $(8 \times 2)$ phase, and its replacement by two large peaks at 0.50 and $0.72 \mathrm{eV}$ is new evidence supporting a metal-insulator (MI) transition. There is no evidence of residual metallic behavior in the $(8 \times 2)$ phase in the mid-IR.

RAS is a powerful technique for probing optically anisotropic surface and interface structure [33], which measures the difference in reflectance, at near normal incidence, of light linearly polarized in two orthogonal directions at the surface plane. The response depends on the imaginary part of the difference in the surface dielectric function in the two orthogonal directions. For these samples, the difference in reflectance parallel and perpendicular to the chains is being measured. The IR data were measured with an InAs detector and the visible-UV data

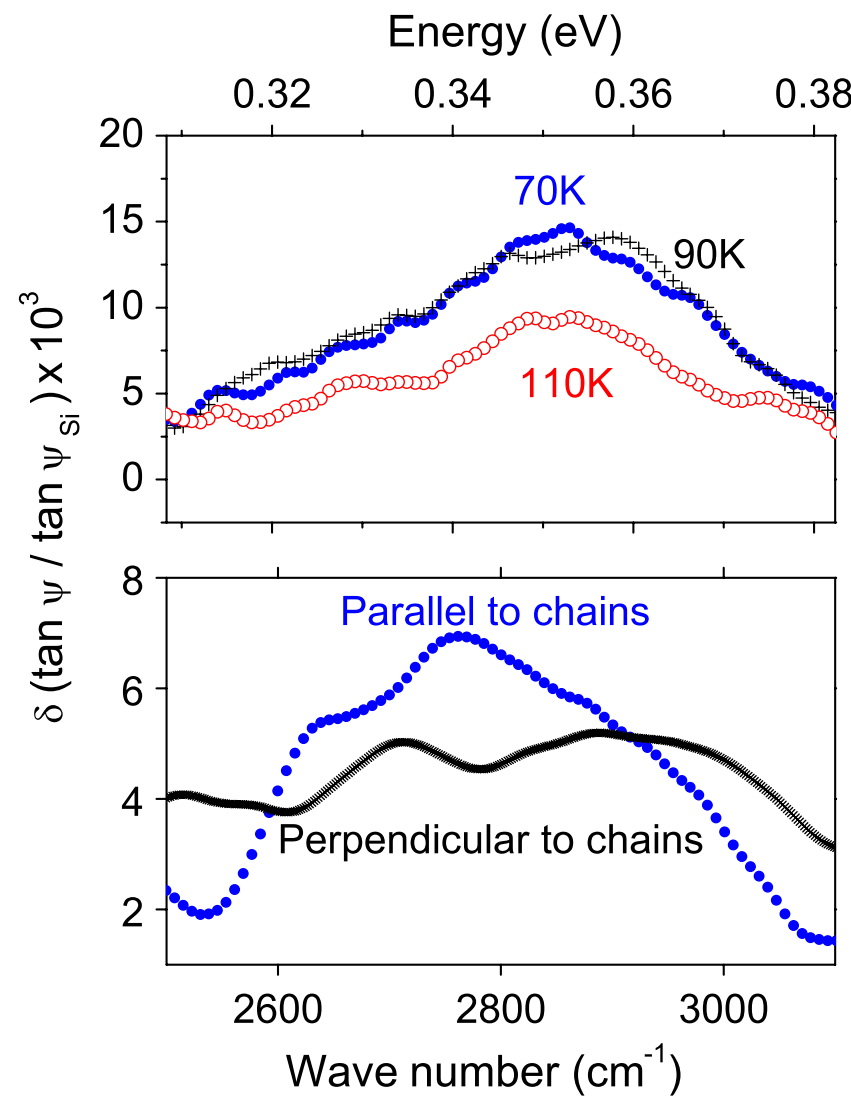

FIG. 3 (color online). Enlarged region for the $(8 \times 2)$ structure from $2500 \mathrm{~cm}^{-1}$ to $3100 \mathrm{~cm}^{-1}$, from which a linearly increasing background has been subtracted. Upper figure: the effect of cooling. Lower figure: the anisotropic response. with InGaAs and Si detectors, the changeover occurring at $0.75 \mathrm{eV}$.

Figure 4 shows measured and calculated RAS spectra. The data reveal a smooth increase to low energy for the RT $(4 \times 1)$ phase, while the LT $(8 \times 2)$ phase shows two sharp positive peaks at 0.50 and $0.72 \mathrm{eV}$ that correspond exactly to the IRSE features discussed above. Positive anisotropy indicates that optical transitions parallel to the chains are dominant in this spectral region. Both phases show the broader, negative $1.9 \mathrm{eV}$ feature, which splits below the MI transition [7,31,32].

The theoretical data are obtained using density functional theory (DFT) within the local density approximation (LDA) for exchange and correlation as implemented in VASP [34]. The RAS is obtained from the slab polarizability in the independent-particle approximation [32] and uses no adjustable parameters. The calculated anisotropy of both the trimer and hexagon models of the $(8 \times 2)$ structure agree well with the experiment above $0.7 \mathrm{eV}$, as has been reported previously [32]. Below $0.7 \mathrm{eV}$, only the hexagon model looks similar to the experimental results. In particular, two positive peaks are predicted, separated by $0.24 \mathrm{eV}$. This splitting agrees very well with the experimental splitting of $0.22 \mathrm{eV}$. The origin of the two peaks in the mid IR can be traced to optical transitions close to the $M$ point of the surface Brillouin zone, indicated by $P_{1}$ and $P_{2} / P_{2}^{\prime}$ in Fig. 5. Around $M$, nearly parallel valence and conduction bands close to the Fermi level give rise to a high joint density of states. From the orbital character of states (not shown here) we can assign $P_{1}$ to transitions between bonding and nonbonding In chain states within the single In zigzag chains, while $P_{2}$ and $P_{2}^{\prime}$ involve in addition In-In bonds between the two parallel zigzag chains. These bonds are exclusively formed for the hexagon model (Fig. 1). The

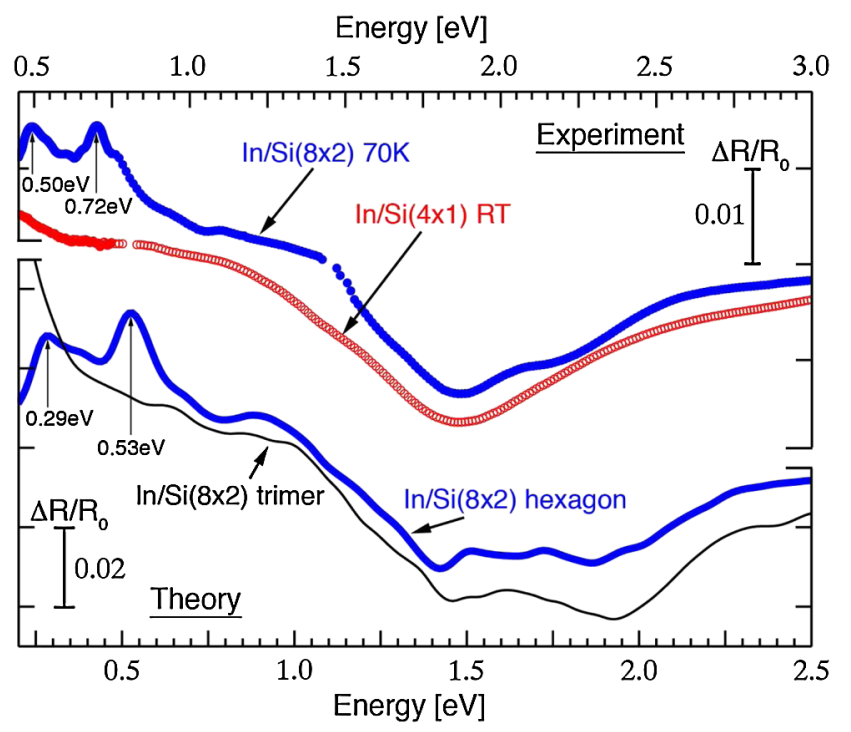

FIG. 4 (color online). RAS spectra of $\mathrm{Si}(111)-(4 \times 1)$-In at RT $(300 \mathrm{~K})$ and $\mathrm{Si}(111)-(8 \times 2)$-In at LT $(70 \mathrm{~K})$ : upper, experiment; lower, theory. Note the different scales. 


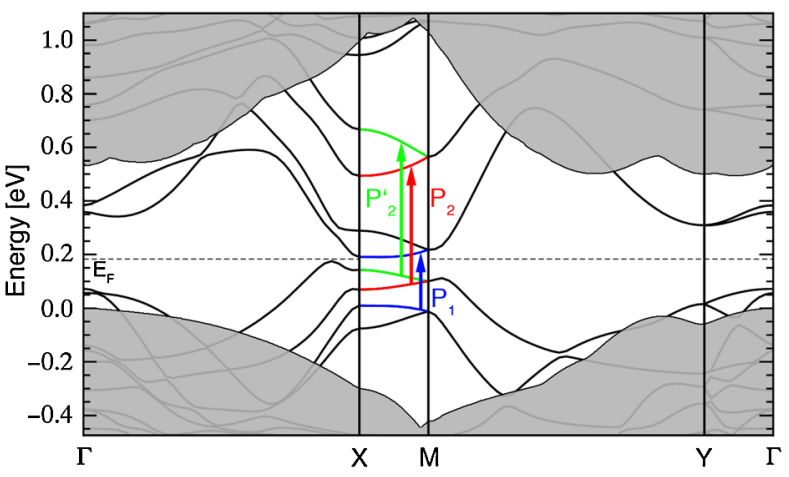

FIG. 5 (color online). Band structure of the hexagon model for $\mathrm{Si}(111)-(8 \times 2)$-In calculated within DFT-LDA. Pronounced optical transitions showing up in the RAS spectra are marked. Gray regions correspond to the projected Si bulk bands. The bulk valence band maximum is chosen as energy zero. The Fermi level is indicated.

spectra measured in the mid-IR are thus directly related to the hexagon structure of the In nanowire array.

Detailed comparison reveals that the calculated mid-IR peaks are redshifted by $0.25 \mathrm{eV}$ (note the different scales in Fig. 4). The underestimation of excitation energies is typical for DFT calculations where self-energy effects are neglected. The complexity and size of the In nanowire structure prevents the calculation of optical spectra using many-body perturbation theory that includes self-energy and excitonic effects [35]. Quasiparticle calculations for the high-symmetry points of the hexagon model surface band structure found self-energy effects to increase the lowest transition energies by $0.26 \mathrm{eV}$ on average [13]. A larger shift of $0.5 \mathrm{eV}$, typical for $\mathrm{Si}$ excitation energies [35], applies to the higher energy negative optical anisotropies, because the optical transitions involve $\mathrm{Si}$ states [32]. Allowing for these energy shifts, the agreement between the calculated and measured RAS spectra is truly impressive.

In conclusion, the first mid-IR optical measurements and $a b$ initio calculations have been reported for the In-Si nanowire system. IRSE has sufficient sensitivity to measure the optically anisotropic surface response of the In chains on the metallic $(4 \times 1)$ surface. The dramatic shift of spectral weight on cooling to form the $(8 \times 2)$ phase is consistent with a MI phase transition. Strong, anisotropic optical interband transitions were found with IRSE and RAS at 0.50 and $0.72 \mathrm{eV}$ for this phase. In contrast to the trimer model, optical response calculations for the hexagon model reproduced the measured features. In particular, the $0.7 \mathrm{eV}$ peak arises from optical transitions involving the additional In-In bonds that are only formed in the hexagon model. These results provide strong evidence in favor of the hexagon model of the In nanowire ground state.

This publication has emanated from research conducted with the financial support of Science Foundation Ireland, DFG, Land Berlin, and the BMBWFT. Supercomputer time provided by the HLRS and the $\mathrm{PC}^{2}$, as well as advice from C. Roland, I. Fischer, A. Roeseler, K. Roodenko, and C. Cobet, is gratefully acknowledged.

*Corresponding author. jmcgilp@tcd.ie

[1] E. H. Lieb and F. Y. Wu, Phys. Rev. Lett. 20, 1445 (1968).

[2] F. J. Himpsel et al., J. Phys. Condens. Matter 13, 11097 (2001).

[3] T. Abukawa et al., Surf. Sci. 325, 33 (1995).

[4] I. G. Hill and A. B. McLean, Phys. Rev. Lett. 82, 2155 (1999).

[5] O. Bunk et al., Phys. Rev. B 59, 12228 (1999).

[6] J.-H. Cho, D.-H. Oh, K. S. Kim, and L. Kleinman, Phys. Rev. B 64, 235302 (2001).

[7] S. Wang, W. Lu, W. G. Schmidt, and J. Bernholc, Phys. Rev. B 68, 035329 (2003).

[8] K. Fleischer et al., Phys. Rev. B 67, 235318 (2003).

[9] J.-H. Cho, J.-Y. Lee, and L. Kleinman, Phys. Rev. B 71, 081310(R) (2005).

[10] X. Lopez-Lozano et al., Phys. Rev. B 73, 035430 (2006).

[11] K. Fleischer et al., Phys. Rev. B 76, 205406 (2007).

[12] T. Kanagawa et al., Phys. Rev. Lett. 91, 036805 (2003).

[13] A. A. Stekolnikov et al., Phys. Rev. Lett. 98, 026105 (2007).

[14] S. Wippermann, N. Koch, and W. G. Schmidt, Phys. Rev. Lett. 100, 106802 (2008).

[15] H. W. Yeom et al., Phys. Rev. Lett. 82, 4898 (1999).

[16] T. Tanikawa, I. Matsuda, T. Kanagawa, and S. Hasegawa, Phys. Rev. Lett. 93, 016801 (2004).

[17] J. R. Ahn et al., Phys. Rev. Lett. 93, 106401 (2004).

[18] S. J. Park et al., Phys. Rev. Lett. 93, 106402 (2004).

[19] J. Guo, G. Lee, and E. W. Plummer, Phys. Rev. Lett. 95, 046102 (2005).

[20] K. Sakamoto, H. Ashima, H. W. Yeom, and W. Uchida, Phys. Rev. B 62, 9923 (2000).

[21] Y. J. Sun et al., Phys. Rev. B 77, 125115 (2008).

[22] T. Uchihashi and U. Ramsperger, Appl. Phys. Lett. 80, 4169 (2002).

[23] H. W. Yeom et al., Phys. Rev. B 65, 241307(R) (2002).

[24] C. Gonzalez, F. Flores, and J. Ortega, Phys. Rev. Lett. 96, 136101 (2006).

[25] J.-H. Cho and J.-Y. Lee, Phys. Rev. B 76, 033405 (2007).

[26] Y. Fukaya, M. Hashimoto, A. Kawasuso, and A. Ichimiya, Surf. Sci. 602, 2448 (2008).

[27] S. Chandola et al., Phys. Status Solidi B 242, 3017 (2005).

[28] K. Fleischer et al., Phys. Rev. B 74, 195432 (2006).

[29] A. Röseler, in Handbook of Ellipsometry, edited by H. G. Tomkins, E. A. Irene, and W. Andrew (Springer-Verlag, Berlin, 2005), p. 763.

[30] K. Hinrichs, M. Gensch, and N. Esser, Appl. Spectrosc. 59, 272A (2005).

[31] K. Fleischer et al., Appl. Surf. Sci. 234, 302 (2004).

[32] S. Wippermann and W. G. Schmidt, Surf. Sci. 603, 247 (2009).

[33] J. McGilp, Prog. Surf. Sci. 49, 1 (1995).

[34] G. Kresse and J. Furthmüller, Comput. Mater. Sci. 6, 15 (1996).

[35] P. H. Hahn, W. G. Schmidt, and F. Bechstedt, Phys. Rev. Lett. 88, 016402 (2001) 